\title{
ARTICLE \\ Hybridized Deep Neural Network Using Adaptive Rain Optimizer Algorithm for Multi-Grade Brain Tumor Classification of MRI Images
}

\author{
V. V. S. Sasank* S. Venkateswarlu \\ Department of Computer Science and Engineering, KoneruLakshmaiah Education Foundation, Vaddeswaram, AP, \\ 522502, India
}

Received: 4 January 2022; Accepted: 18 January 2022; Published: 19 January 2022

\begin{abstract}
Classification of brain tumor is highly significant in the medical field in real-world to improve the progress of treatments. The seriousness behind the tumors are normally graded based on the size into grade I, grade II, grade III and grade IV. This is where the process of multi-grade brain tumor classification gains attention. Thus, the article focusses on classifying the brain MRI images into four different grades by proposing a novel and a very efficient classification strategy with high accuracy. The acquired images are pre-processed with the help of an Extended Adaptive Wiener Filter (EAWF) and then segmented using the piecewise Fuzzy C- means Clustering (piFCM) technique. Then the most ideal features such as the texture, intensity and shape features that can best explain the growth of tumors are extracted using the Local Binary Pattern (LBP) and the Hybrid Local Directional Pattern with Gabor Filter (HLDP-GF) techniques. After extracting the ideal features, the Manta Ray Foraging Optimization (MRFO) method has been introduced to optimally select the most relevant features. Finally, a Hybrid Deep Neural Network with Adaptive Rain Optimizer Algorithm (HDNN- AROA) is proposed to classify the grades of brain tumors with high accuracy and efficiency. The proposed technique has been compared with the existing state-of-the-art techniques relevant to brain tumor classification in terms of accuracy, precision, recall and dice similarity coefficient to prove the overall efficiency of the system.
\end{abstract}

Keywords: Multi-grade brain tumor classification, Segmentation, Feature extraction, Feature selection, Classification

\section{Introduction}

Diseases associated with the brain are evolving as a big issue in modern society specifically malignant brain tumors which seriously affect the human lives ${ }^{[1]}$. In brain tumor disease, Gliomas are considered as highly occurring malignant brain tumor that is caused by the abnormal transformation of brain cells also, it is basically classified into Low-Grade Gliomas (LGG) and High-Grade Gliomas (HGG) ${ }^{[2]}$. Growth of HGG is highly worsened also considered as malignant tumors and need surgery due to its complex nature. Whereas, LGG has low influences in human life and the life time of the patient is extended over the treatment ${ }^{[3]}$. These tumors are differentiated

\footnotetext{
*Corresponding Author:

V. V. S. Sasank,

Department of Computer Science and Engineering, KoneruLakshmaiah Education Foundation, Vaddeswaram, AP, 522502, India; Email: sasank64@gmail.com
} 
by various techniques like X-ray, Single-Photon Emission Computed Tomography (SPECT), Ultrasound, Magnetic Resonance Imaging (MRI), Positron Emission Tomography (PET), Magnetic Brain Wave Graph (MEG), Computed Tomography (CT), and Electroencephalogram $(\mathrm{EEG})^{[4-6]}$.

The exact size and volume of the malignant tumor is accurately identified by using MRI scan and this MRI technique is considered as the most widely used comprehensive method than any other medical imaging techniques ${ }^{[7]}$. In addition to that, historical screening is done to perform grade labelling in images using MRI. Biopsy is a method which is required by this invasive labelling technique. 3 Tesla (3T) MRI is a non-invasive method developed to create a robust in alternative for biopsy ${ }^{[8,25]}$. Without a doubt, grade estimation in brain tumor is performed by a MRI screening method that provides a large amount of information to the radiologists and neuro-surgeons. For instance, anatomical sequences can be related with perfusion and diffusion imaging for an improved analysis. To advance molecular facts of cancerous brain substances, MR Spectroscopy (MRS) can be used as additional technique. This diversity of acquirements brands MRI a multimodal device for tumor oncology ${ }^{[9,10]}$.

With the significant efforts, automatic identification and segmentation of irregularities is still a great challenge because of the inadequacies in image acquisition, scanner settings variation, variations in the anatomy of brain morphology and pathological variations in appearance ${ }^{[11,26]}$. Early stage detection of brain tumor position, recognition and classification is considered as a serious problem in medical science. When compared to the previous conventional machine learning methods, deep learning methods have accuracy to detect the tumor in brain MRI over the large amount of datasets ${ }^{[12,27]}$. This kind of enhancement helps the physicians to observe and track the occurrence and growth of tumor-affected areas at various stages so that, they can offer proper analysis with these image scanning ${ }^{[13,28,29]}$.

Overall, key issue in the multi-grade tumor identification is early stage detection which helps to treat people with proper treatment. Treatments like radiation, therapy, surgery or chemotherapy can be initiated by the physicians to cure the disease. When the tumor is detected in its early stage, it is obvious that the survival chances of tumor-infected patient can be increased significantly. In recent years, there has been a proliferation of machine and especially deep learning techniques in the medical imaging field ${ }^{[14,30,31]}$. Deep learning algorithms also referred to as deep neural networks, are built using large stacks of individual artificial neurons, each of which performs primitive mathematical operations of multiplication, summation, and thresholding. One of the key reasons for the success of these modern deep neural networks is the idea of representation learning; the process of learning useful features automatically from the data as opposite to manual selection by expert humans ${ }^{[15,32]}$.

Multi-grade tumor classification is highly significant to determine the growth and stage of the tumor inside the brain. To reduce the mortality rate due to brain tumors and to improve the quality of life for every patients, an efficient and highly accurate classification strategy is much required. A descent classification strategy with reduced error can easily identify the rate of growth of the tumor as well as helps in detailed analysis of the tumors paving way for efficient remedies. There are a vast count of techniques available to categorize the tumors identified as harmful and harmless whereas the technological side still lags in obtaining an efficient and accurate classification results based on the stages (grades) of the tumors. The immediate requirement of a powerful classification strategy and the lag in the research side in accurate identification of the grade of tumors motivated to propose this work. The major objective of the proposed work is to accurately classify the brain tumors based on the grades. The chosen neural network is highly efficient in classifying the tumors based on the growth and the accuracy of it has been further improved with the hybridization of an efficient optimization algorithm that converges better improving the learning rate. The major contributions of the proposed work include the following:

$>$ A novel hybrid Local Directional Pattern with Gabor filter (HLDP-GF) along with Local Binary Pattern (LBP) has been proposed for extracting the ideal features related to texture, intensity and shape of the images.

$>$ An efficient Manta Ray Foraging Optimization (MRFO) is proposed for selecting the most relevant features with the elimination of high dimensionalities to improve the classification performance and to reduce the time complexity.

$>$ The accuracy in the classification of brain MRI images based on grades has been drastically improved using the proposed Hybrid Deep Neural Network with Adaptive Rain Optimizer Algorithm (HDNN-AROA) with better convergence.

$>$ The performance of the proposed approach has been evaluated in terms of accuracy, precision, recall, and dice similarity to prove its efficiency against other existing state-of-the-art techniques.

The remaining sections of the paper are organized as follows: Sections 2 provides the survey of recently 
published papers relevant to brain tumor classification. Section 3 presents the proposed methodologies. Section 4 demonstrates the simulation part along with results and discussion. Section 5 provides the conclusion and future scopes of the proposed article.

\section{Literature Review}

Brain tumor causes significant damage to health and leads to death due to its severe nervous system disorder. For the treatment and analysis of glioma, MRI becomes a most widely used principle diagnostic system. In brain tumor MRI, segmentation and classification process is considered as a complex task. So, C. Narmatha et al. ${ }^{[16]}$ developed a fuzzy brain-storm optimization method for brain tumor segmentation and classification. Cluster center calculation was determined by brain-storm optimization algorithm and it provides them the highest priority. Optimal network structure was obtained by performing several iteration of fuzzy algorithm. In terms of various results, brain storm algorithm outperforms existing methods.

Nowadays, automatic diagnosis of brain tumor detection is contemplated as an important research topic. Due to the various characteristics of tumors, it is very difficult to classify the multi-tumor brain image classification. Neurologists or neurosurgeons are assisted by the recent deep neural networks which normally classify the tumors in an efficient manner. But, deep learning algorithms are affected by overfitting and vanishing gradient problem. In this research ${ }^{[17]}, \mathrm{R}$. Lokesh Kumar et al. suggested a deep network model named as ResNet-50 which solve the overfitting and vanishing gradient problems. A three-tumor brain MRI dataset consisting of 3064 images were utilized to estimate the efficiency of the proposed method.

Generally, cancer has created the lowest survival rate in adults and children around the world. Wrong classification can lead to bad sequences in people lives. For correct treatment, it is very essential to recognize the correct category and grade of humor in early stage. In addition to that, manual detection of tumor grade is more time consuming and may lead to human error thanks to the huge amount of data as well as several kinds of brain tumor types. So, it requires automatic detection using computer assisted diagnosis (CAD). In this research ${ }^{[18]}$, Wadhah Ayadi et al. deep convolution neural network (CNN) is developed to classify the tumor in an accurate manner. This deep CNN model is evaluated against three different kinds of datasets.

Benign and malignant are two different types of brain tumor in which both types have different properties.
Henceforth, it is very essential to segment and classify two different kinds of tumor from MRI so that, doctors can easily provide a correct treatment to the patient. In this research ${ }^{[19]}$, V.V. Sasank and S. Venkateswarlu developed a machine learning based classification in brain MRI. In addition to that, pre-processing was done with the help of Contrast Limited Adaptive Histogram Equalization (CLAHE) as well as Laplacian of Gaussian (LoG) methods. After that feature extraction was performed and optimization based method was applied to select the correct features from huge number of features. Finally, classification of brain tumor was performed by proposed Kernel based Softplus Extreme Learning Machine (KSELM) technique. Different types of dataset like BRATS 2014, 2015, 2018 and BRT (Brain Tumor) were taken to evaluate the proposed methodology.

In the field of medical imaging analysis, classification of brain tumor grade is considered as an essential process to assist the neuro-surgeons during clinical analysis. Hiba Mzoughi et al. ${ }^{[20]}$ developed a deep multi-scale 3D CNN for MRI gliomas brain tumor classification. This algorithm efficiently classified the glioma brain tumor into high grade gliomas (HCG) and low grade gliomas (LCG). The proposed method had the possibility to join both global as well as local contextual data with reduced weights over the small kernels in 3D convolutional layer and a deep network. In addition to that, intensity normalization and adaptive contrast enhancement techniques were applied to perform the pre-processing stage in proposed methodology. BRATS 2018 dataset was utilized to evaluate the proposed methodology in which it creates discriminative feature map to differentiate the LCG and HCG variants.

From the surveys taken, it has been identified that the available strategies for brain tumor classification is not so accurate and efficient. Moreover, the learning time of the classifiers are too high when the classification is performed formultiple images with large quantity of features. To compromise the immediate requirement of an accurate and efficient classification strategy, the article has been proposed with the most efficient techniques known so far.Selection of optimization techniques influences the results of the proposed technique and hence the most efficient algorithms with high convergence rate are chosen for generating accurate classification results. The use of AROA algorithm efficiently reduced the computational complexity of the learning process with minimized error. The main aim of the proposed article is to improve the classification accuracy along with reduced time complexity. 


\section{Proposed Methodology}

The article proposes a hybrid framework for multigrade brain tumor classification to classify the grade of brain tumorsaccurately. The brain tumor images are initially acquired,and the pre-processing steps are introduced to remove the noise present in it. The EAWF filter has been utilized with the aid of removing the unwanted noise present in the image as well as to improve the quality of the image. After the pre-processing step, the images are subjected to segmentation, where the piFCM technique has been employed to efficiently segment the images based on the image features applicable in the domain of real-world applications. The main aim in using the piFCM clustering technique is to reduce the uncertainty arising while clustering the images employinga powerful uncertainty absorbent.

Succeeding the clustering process, feature extraction is carried out on the segmented images using ULBP, LDP and Gabor filter. In some cases, the extracted features might cover both relevant and irrelevant features in large dimensions. Thus an efficient feature selection method has been introduced to identify the required and sufficient features for classification. The proposed technique presents an efficient optimization algorithm called MRFO for optimal feature selection from the extracted set of features. The selected features are given as input to the classification phase where the tumors get classified into different grades. For classification, an efficient HDNNAROA has been proposed to provide better prediction results with high accuracy. Figure 1 depicts the proposed framework for multi-grade brain tumor classification.

\subsection{Pre-processing}

Pre-processing is the initial step followed in the proposed work to improve the image quality by making it sharper and to remove the undesirable noise present in the image. The images acquired from the databases like Radiopaedia dataset and brain tumor datasetconsists of MRI brain tumor images that are labelled into four grades based on the tumor's intensity and growth. Usually, the images of MRI consists of noise owing to various factors like sensors, temperature, lighting levels, granularity, etc. These images are pre-processed using the EAWF ${ }^{[21]}$ filter to improve the clarity of the images by removing the noise while preserving the edge information of the images. To improve the original adaptive wiener filter, a dispersion index has beenintroduced in place of variance. This helps in identifying the clustered and dispersed occurrences.

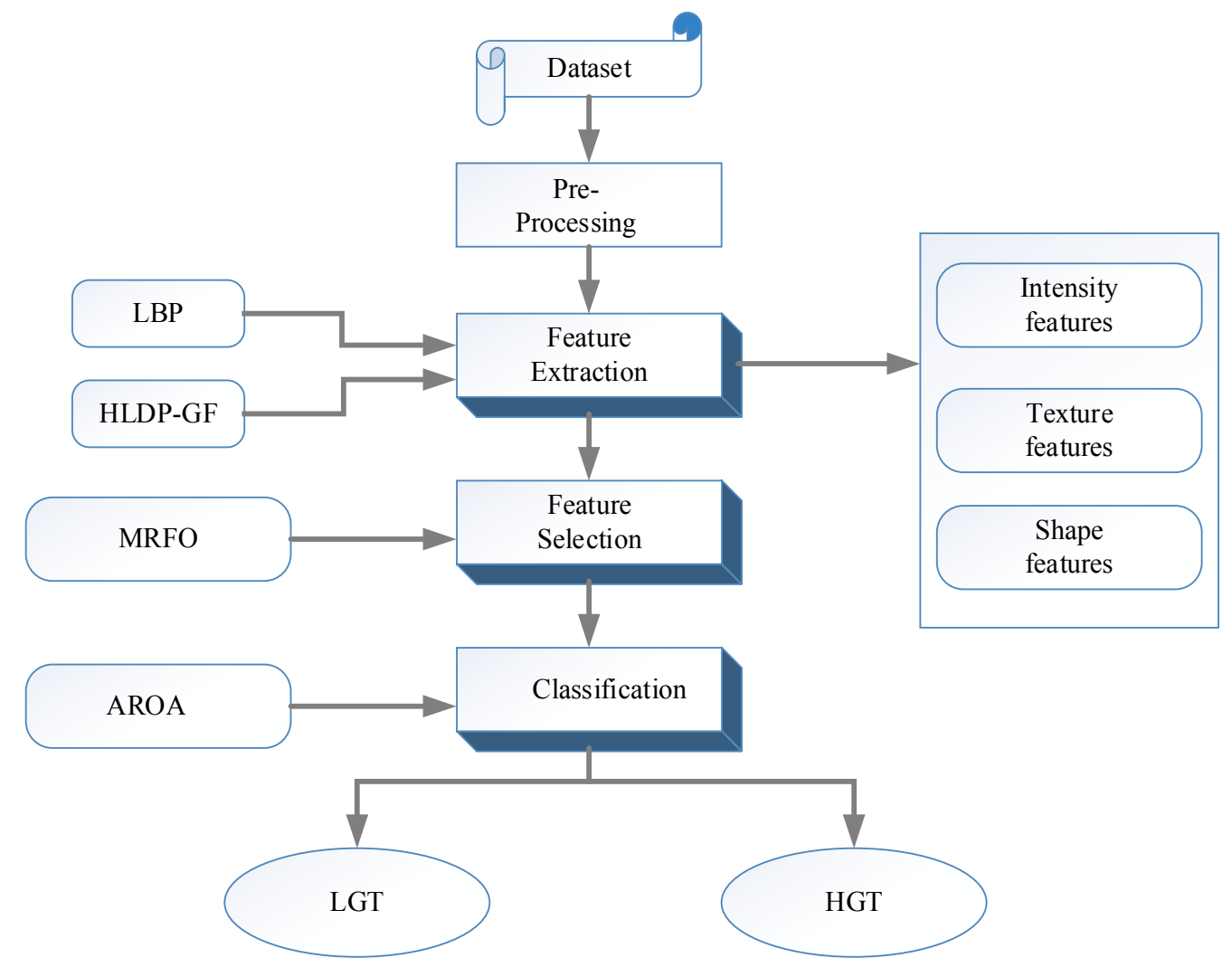

Figure 1. Proposed Framework for Multi-Grade Brain Tumor Classification 
Normally the pre-processing strategy eliminates the noise present in the image. The use of EAWF filter reduces any kind of noise identified in the image along with improving the image quality. Considering a pixel location $p_{1}, p_{2}$ of an input image $I_{p}$, the normal AWF determines the noise using the mean and noise variance. The extended technique uses a dispersion index to identify the noise approximately from the image. The additive noises present in the image are retarded to smoothen the image with high pixel quality. Dispersion index can be identified as:

$d_{i}=\frac{\sigma^{2}}{\mu}$

Applying the dispersion index onto the normal AWF filter, the equation gets modified like the following:

$W_{f}\left[I_{p}\left(p_{1}, p_{2}\right)\right]=\mu+\frac{d_{i}-\sigma_{n}^{2}}{d_{i}}\left[I_{p}\left(p_{1}, p_{2}\right)-\mu\right]$

Upon simplification, the overall output after preprocessing can be identified through the following formula:

$W_{f}\left[I_{p}\left(p_{1}, p_{2}\right)\right]=I_{p}\left(p_{1}, p_{2}\right)-\mu\left(\frac{\sigma_{n}^{2}}{\sigma^{2}}\left[I_{p}\left(p_{1}, p_{2}\right)-\mu\right]\right)$

Where $\mu$ indicates the mean value and $\sigma_{n}$ indicates the variance of noise.

From Equation (3), the enhanced quality of the input image can be identified. The noise present in the image can be efficiently removed using the improved filter. The noise variance indicates the noise present in the image and the value obtained for the noise variance demonstrates the influence it caused to the image. Reducing the noise improves the image quality and provides an image that is more clear and visible than the original image.

The major benefit of using this improved filter is that the speckle noise present in the image will be keenly removed. Speckle noise is seen mostly in the MRI images and it occurs possibly due to environmental conditions. This noise has a direct impact on the contrast and texture of the images as it gives a grainy appearance and hence removing this noise is highly significant. AWF filter is popularly known to remove the additive noise identified in the images and the improved AWF filter removes the speckle noise more efficiently, meanwhile improving the image quality.

\subsection{Image Segmentation}

Succeeding the pre-processing step, the segmentation step through clustering has been carried out. The preprocessed MRI images obtained are subjected to segmentation based on the piecewise Fuzzy C-means Clustering technique (piFCM).This technique has been employed for clustering as the data objects that are to be segmented might be connected with more than one clusters with diverse memberships, and this technique can efficiently cluster such objects exhibiting relationships between clusters. Segmentation prevails as a major task as it clusters the images into groups based on the features which makes the extraction process simpler.

Normal fuzzy clustering clusters the images based on the membership between the image clusters. The piecewise image centroid are identified to compute the segmented parts using the piFCM. The identified centroid in the object determines the shortest distance between the segmented images to identify the membership among various parts of the object. In this way, the images are segmented identifying the relationships between them.

Initially, to cluster the images, the data points in the images are considered as a fuzzy set determining the relationships between each other. The fuzzy clustering technique is followed by almost all the segmentation techniques in image processing systems but the difficulty lies in identifying the data objects and cluster centroids from the distance computation. The distance formulation of the standard fuzzy clustering can be given as $\sum_{c=1}^{r} f\left(\mu_{k}^{c}, q_{l}^{c}\right)$. The formula denotes the distance between $p_{k}$ and $q_{l}$ where $c$ denotes the count of pieces that are segmented. From Equation (4), the centroids and the data objects are difficult to identify and hence piecewise FCM is adopted to overcome this issue with the introduction of multi-membership function. Let the MRI brain image dataset be represented as $D=\left\{d_{1}, d_{2}, \ldots . d_{n}\right\}$ which is a multi-membership dataset imitated from a Bootstrap Probability (BP) set and the $k^{\text {th }}$ data object in the dataset can be represented as:

$d_{k}=\left(\mu_{k}^{1}, \ldots . . \mu_{k}^{i}, \ldots . . \mu_{k}^{r}\right)$

The data object identified through Equation (5) aligns the membership degree of the identified object to all the clusters obtained from the available BPs. Thus the vector $d_{k}$ is a $\left(\sum_{c=1}^{r} L_{c}\right)$ dimensional vector where $\left\|d_{k}\right\|_{1}=r \forall k$. The centroid for the clustering can be computed using the piFCM technique and can be represented as:

$q_{l}=\left(q_{l}^{1}, \ldots . q_{l}^{c}, \ldots . q_{l}^{r}\right)$

The centroid that are identified through Equation (6) is also a vector and the vector dimension for $q_{l}$ can 
be identified as $\left(\sum_{c=1}^{r} L_{c}\right)$ casing $_{r}$ pieces. Every centroid computed through Equation (6) is known to be the piecewise centroid as the centroid iscomputed for the pieces segmented from the image. Thus $q_{l}$ can be termed as the piecewise centroid for the dataset D for fuzzy clustering. Considering the fuzzy clustering in terms of multi-membership data, the distance between the centroid and the data object can be given as:

$\operatorname{dis}\left(d_{k}, q_{l}\right)=\sum_{c=1}^{r} f\left(\mu_{k}^{c}, q_{l}^{c}\right) \quad \forall k, l$

The distance computed is the Euclidean distance between the centroid and the respective data object and the minimization of the distance between them will provide clusters that are more relevant.

The brain MRI images are mostly uncertain, and reducing the uncertainty of the images are essential while passing on values to the membership functions. Uncertainties reduce the efficiency of clustering and the relevance between the data objects cannot be identified accurately due to the presence of various kinds of uncertainties. Some types are present in the input image itself and many others might get added later. To obtain an efficient clustering output and to improve the classification with high accuracy, the uncertainties are required to be reduced using a suitable uncertainty absorbent. With the use of these absorbents, the uncertainty present in every image can be reduced and the clustering becomes more relevant and efficient. To eliminate the uncertainty present in the image, a parameter to reduce the uncertainty can be added to every pixel values subjected to the membership function. This parameter can be termed as the hesitation degree denoted as follows:

$\pi_{f}(d)=1-\mu_{f}(d)-q_{f}(d)$

In the above equation, $\mu_{f}(d)$ and $q_{f}(d)$ symbolizes the multi-membership function and the centroid for the data objects respectively. Therefore, the piFCM clustering function along with the uncertainty absorbent can be given as follows:

$$
F=\left\{\mu_{f}(d), q_{f}(d), \pi_{f}(d)\right\} \quad \forall d \in D
$$

From the above Equation (8) the clusters that are segmented can be identified along with the negation of uncertainty. The piFCM clustering mechanism enhances the segmentation and the ideal clusters are obtained without uncertainties through the use of the uncertainty absorbent.

\subsection{Feature Extraction}

Feature extraction is one of the most significant functionality followed in the proposed article for brain tumor classification. This process indicates the collection of most required information from the images to get a deep idea about the image and to obtain an accurate output in classification. Any kind of data objects are identified or get classified only based on the features associated with the data objects and the learning techniques highly relies on the features of the image to classify more efficiently. Extraction of relevant and most important features related to shape and texture paves way for efficient classification of the brain images. The clustered images from the segmentation process are subjected to the feature extraction phase where the most important features are identified based on the clusters itself. The article proposes a uniform local binary pattern (ULBP) and hybrid local directional pattern with gabor filter (HLDP-GF) to extract the required features. The features like texture, shape and intensity signifies the image with keen contrast. Extracting these features enable the identification of tumor based on the grades more accurately.

The LBP features related to textureare initially extracted and then the hybrid LDP with Gabor filter is introduced to extract the left out features that constitutes the intensity and shape features of the image. Moreover, the edge information of the images are preserved through ULBP and if the features identified in the edge of the images are found significant. Those are extracted. Then the LDP is applied to extract the intensity features related to the brain image. Intensity features like mean, variance, standard deviation, skewness, kurtosis, etc., constitute the major part of the image and identifying and extracting these features will improve the classification accuracy with best results. Finally, the shape features are extracted using the Gabor filter. After the extraction of texture and intensity features, the same images are then given as an input to the gabor filter where the most important shape features like shape index, irregularity, perimeter, area and circularity are extracted.

\section{ULBP based Feature Extraction}

This phase constitutes the major part in extracting the texture based features. This is highly significant and the major features are extracted on the basis of a pattern. Initially, the histogram is constructed for the image and then the features are identified through the circular domains used in the histogram. The ULBP pattern associates almost all the required texture patterns of the image and the information identified in the edges, end points, isolated points, corners, etc. are extracted more efficiently. This method is highly robust and ensures efficient scaling of the images with simplicity. Using 
ULBP, the uniform patterns are recognized through merging of $2^{l}$ uniform patterns with $(L+1)$ uniform and non-uniform patterns where the spatial transitions will not be greater than 2 and the calculation for ULBP can be identified using the following equation:

$U_{L B P}(l, m)= \begin{cases}\sum_{l=0}^{L-1} p\left(b_{l}-b_{c}\right), f \quad U \leq 2 \\ L+1, \quad \text { otherwise }\end{cases}$

The computation of the ULBP can be formulated as follows:

$$
\begin{aligned}
& U=\left|p\left(b_{L-1}-b_{c}\right)-p\left(b_{o}-b_{c}\right)\right|+ \\
& \sum_{l=1}^{l-1}\left|p\left(b_{l}-b_{c}\right)-p\left(b_{l-1}-b_{c}\right)\right| \\
& p(x)= \begin{cases}1 ; & \text { iff } x \geq 0 \\
0 ; & \text { otherwise }\end{cases}
\end{aligned}
$$

In the above equation, $U_{L B P}(l, m)$ represents the ULBP computation of a circular domain with $l$ pixels of radius $m$. $b_{l}(l=1,2, \ldots . l-1)$ represents the pixel values identified in the neighborhood of the circular domain and $b_{c}$ is the central gray value of the pixel in the circular domain.

The ULBP operator is more efficient than the standard LBP operator due to its simple computation and due to its capability in identifying only the required information thus reducing the storage requirements.

Along with the ULBP features, some of the common and most important texture features ${ }^{[22,24]}$ are extracted such as energy, correlation, contrast, dissimilarity and homogeneity are indicated as the following:

$$
\begin{aligned}
& \text { Energy } \quad e_{n}=\sqrt{\sum_{j=0}^{a-1} \sum_{k=0}^{d-1} f^{2}(j, k)} \\
& \text { Correlation } \quad c_{r}=\frac{\sum_{j=0}^{a-1} \sum_{k=0}^{d-1}(j, k) f(j, k)-M_{j} M_{k}}{\sigma_{x} \sigma_{y}} \\
& \text { Contrast } \quad c_{t}=\sum_{j=0}^{a-1} \sum_{k=0}^{d-1}(j-k)^{2} f(j, k) \\
& \text { Dissimilarity } \quad d_{s}=\sum_{j=0}^{a-1} \sum_{k=0}^{d-1}(|j-k| f(j, k)) \\
& \text { Homogeneity } \quad h_{m}=\sum_{j=0}^{a-1} \sum_{k=0}^{d-1}\left(\frac{1}{1+(j-k)^{2}} f(j, k)\right)
\end{aligned}
$$

Thus the texture feature ULBP together with other extracted texture features can be indicates as a vector $F_{1}$ as follows:

$$
F_{1}=\left[U_{L B P},\left(e_{n}\right),\left(c_{r}\right),\left(c_{t}\right),\left(d_{s}\right)\left(h_{m}\right)\right]
$$

\section{HLDP-GF based Feature Extraction}

The LDP is used to identify the intensity features present in the input MRI image $(h, v)$ through the use of the Kirsch masks that can recognize the pixel values from all the eight directions. Initially, a binary code holding eight bits is assigned to every pixel of the input image. The Kirsch mask is applied in eight different orientations to obtain the information present in the edges. This technique highly preserves the edge information andthe relevant intensity features identified in the edges of the images are extracted. The LDP feature for the image segment can be identified as follows:

$L_{D P}(h, v)=\sum_{m=0}^{7} f\left(K_{m}-K_{A}\right) 2^{m} ; f(x)=\left\{\begin{array}{l}1 ; \text { iff } x \geq 0 \\ 0 ; \text { otherwise }\end{array}\right.$

From the above equation, $(h, v)$ indicates the input segmented image subjected to feature extraction, $K_{m}$ indicates the Kirsch mask and $K_{A}$ indicates the Kirsch activation function.

The required LDP codes are calculated for all the available pixels of the input images and then the input image (I) can be represented using the histogram as:

$H_{g}(I)=\sum_{h=1}^{M} \sum_{v=1}^{N}\left(L_{D P}(h, v), I\right) ; \quad f(x, I)=\left\{\begin{array}{l}1 ; x=I \\ 0 ; \text { otherwise }\end{array}\right.$

Along with the LDP features, some of the other important intensity features are extracted such as mean, standard deviation, skewness and kurtosis which are formulated as:

$$
\text { Mean } \quad M=\left(\frac{1}{a^{*} d}\right) \sum_{j=0}^{a-1} \sum_{k=0}^{d-1} f(j, k)
$$

Standard Deviation $S_{D}=\sqrt{\frac{1}{(a * d)} \sum_{j=0}^{a-1} \sum_{k=0}^{d-1}(f(j, k)-M)^{2}}$

Skewness $S_{K}=\left(\frac{1}{a^{*} d}\right) \frac{\sum_{j=0}^{a-1} \sum_{k=0}^{d-1}(f(j, k)-M)^{3}}{S_{D}{ }^{3}}$

Kurtosis $K_{R}=\left(\frac{1}{a^{*} d}\right) \frac{\sum_{j=0}^{a-1} \sum_{k=0}^{d-1}(f(j, k)-M)^{4}}{S_{D}{ }^{4}}$

All the above mentioned features are some examples of the intensity features considered in this article. The gabor filter is introduced in combination with the LDP to enhance the feature extraction process. The features that are required to be extracted using the gabor filter are the shape features such as area, shape index, circularity, perimeter, irregularity, etc. But extracting these features only through gabor filter holds a huge amount of high dimensional and redundant information. Thus the gabor filter is combined with the LDP to obtain the shape features along with the intensity features.

Initially, the gabor filter is applied to the segmented 
MRI images to obtain the feature graphs and then the histograms for the pixels are constructed through the use of LDP operator mentioned in (18). The SFG (Superimposed Feature Graph) of the gabor filter defines the gabor features relevant to the shape and the graphs are constructed with the help of the identified shape features. After extraction the histograms are defined only for the identified pixels through the SFG where the above mentioned intensity features are extracted. The Gabor filter for a pixel can be represented as follows:

$G_{\alpha, \beta}(h, v)=\frac{\left\|w_{\alpha, \beta}\right\|}{\sigma^{2}} e^{\frac{\left\|w_{\alpha, \beta}\right\|^{2}\|z\|^{2}}{2 \sigma^{2}}}\left[e^{\left(i_{\alpha, w^{2}}\right)}-e^{\frac{-\sigma^{2}}{2}}\right]$

In the above equation, $\alpha$ defines the orientation and $\beta$ defines the scale of gabor filter, $\sigma$ defines the radius of the envelope determined by Gaussian function, $z=(h, v)$ and $w_{\alpha, \beta}$ defines the wave vector. The convolution operation to represent the gabor filter for the input image can be given as:

$$
C_{\alpha, \beta}(h, v)=I(h, v) * G_{\alpha, \beta}(h, v)
$$

The magnitude value for the gabor filter is computed that corresponds to different response values for every pixel position of the image. The magnitude value corresponding to the gabor filter can be identified using the following equation:

$$
\mathrm{M}_{\alpha, \beta}(h, v)=\sqrt{R_{e}\left(C_{\alpha, \beta}(h, v)\right)^{2}+I_{m}\left(C_{\alpha, \beta}(h, v)\right)^{2}}
$$

The other major shape features ${ }^{[23]}$ of the image along with area and perimeter extracted are as follows:

$$
\text { Centroid } c_{r}=\left(x_{1}, y_{1}\right) ; x_{1}=\frac{l_{10}}{l_{00}} ; y_{1}=\frac{l_{01}}{l_{00}}
$$

Length of major axis

$$
Q=2\left[\frac{2\left(\gamma_{20}+\gamma_{02}+\sqrt{\left(\gamma_{20}-\gamma_{02}\right)^{2}+4 \gamma_{11}^{2}}\right)}{\gamma_{00}}\right]^{\frac{1}{2}}
$$

Length of minor axis

$$
R=2\left[\frac{2\left(\gamma_{D}+\gamma_{Q}-\sqrt{\left(\gamma_{D}-\gamma_{Q}\right)^{2}+4 \gamma_{1}^{2}}\right)}{\gamma_{0}}\right]^{\frac{1}{2}}
$$

\section{Central Moments}

$$
\gamma_{p q}=\sum_{h} \sum_{v}\left(h-x_{1}\right)^{p}\left(v-y_{1}\right)^{q} f(h, v)
$$

$$
\text { Image Moments } i m_{p q}=\sum_{h} \sum_{v} h^{p} v^{q} f(h, v)
$$

The above features are the features corresponding to the shape of the image. The LDP operator works on finding the histogram for the feature graphs constructed by the Gabor filter to extract the shape features. The output generated from the histogram will be the required intensity features. The hybrid form representation to extract the shape and intensity features can be given as follows:

$\psi_{L_{\mathbb{B}} G}=\sum_{m=0}^{7} f\left(G_{m}-G\right) 2^{m}$

After the feature graph construction, the histogram operator works on building histograms on the graphs to identify the edge features and to extract them. The histogram operator to construct the histogram on pixels can be modified as:

$H_{g}(I)=\sum_{h=1}^{M} \sum_{v=1}^{N} \sum_{m=0}^{7}\left[\left(f\left(G_{m}-G\right) 2^{m}\right), I(h, v)\right]$

Thus the features extracted through the HLDP-GF along with other intensity and shape features can be represented as a vector as follows:

$F_{2}=\left[\psi_{L_{D P} G},(M),\left(S_{D}\right),\left(S_{K}\right),\left(K_{R}\right),\left(c_{r}\right),(Q),(R),\left(\gamma_{p q}\right),\left(i m_{p q}\right)\right]$

Therefore, the overall features that are extracted from the MRI brain image can be obtained by adding the feature vectors in (16) and (35). This is described as:

$$
\begin{aligned}
F=[ & \left\{\left(U_{L B P}\right)\left(e_{n}\right),\left(c_{r}\right),\left(c_{t}\right),\left(d_{s}\right)\left(h_{m}\right)\right\},\left\{\left(\psi_{L_{D P} G}\right),(M),\left(S_{D}\right),\right. \\
& \left.\left.\left(S_{k}\right),\left(K_{R}\right),\left(c_{r}\right),(Q),(R),\left(\gamma_{p q}\right),\left(i m_{p q}\right)\right\}\right]
\end{aligned}
$$

\subsection{Feature Selection Using MRFO}

Feature selection is the prime segment in the proposed brain tumor multi-grade classification approach. The feature selection process indicates the selection of best features from the identified set of features. For the feature selection process, this article proposes the MRFO optimization algorithm mimicking the foraging process of manta rays. These are marine creatures popularly known to be one of the largest known sea animal. These creatures follow a very efficient foraging strategy to identify and eat the sea planktons found in the ocean. The three unique and intelligent foraging strategies of manta rays can be identified as follows:

Chain foraging:In this foraging strategy, group of individuals move towards the food following a lead. Also, the individuals find the food that are missed by the lead and hence best food are also identified by every individual in the group.

Cyclone Foraging:The group of individuals in this foraging strategy make a spiral movement towards the patch of food recognized. Along with following the lead in a spiral pattern, each individual in the group forages separately along the path. 
Somersault Foraging:The angle of the identified food is represented as a central point and every individual of the group somersaults to reach the food and thus the positions of each individual are updated frequently.

The MRFO can be imitated into the proposed technique for feature selection by taking the features extracted through LBP and HLDP-GF as the individuals in the search space. The optimal features in the group are selected based on the fitness function that signifies the tumor based on the grade.Initially, the group of features (n) extracted are represented as the individuals in the D-dimensional search space in matrix formulation. It can be described as follows:

$F_{S}=\left[\begin{array}{ccc}f_{1,1} & f_{1,2} \ldots \ldots . f_{1, d} \\ f_{2,1} & f_{2,2} \ldots \ldots . f_{2, d} \\ \ldots & \ldots \ldots & \ldots . \\ \ldots & \ldots . . & \ldots . \\ f_{n, 1} & f_{n, 2} \ldots \ldots . & f_{n, d}\end{array}\right]$

The random placement of features signifies that there is no experience inthe fitness evaluation and the optimal features are unknown. Therefore, an objective function is formulated to evaluate the initial placement. For this purpose, the minimization of the features is enclosed to shorten the amount of features and to discard the features with greater dimensions. The error in classification of the features into optimal and worst influences the feature selection process and hence it is embedded in the fitness evaluation function as follows:

Fit $=\Omega * \Psi+(1-\Omega) * \frac{|f|}{|F|}$

In the fitness evaluation, $f$ resembles the count of features extracted in the feature extraction process, $F$ resembles the total count of features identified in the dataset, $\Psi$ symbolizes the classification error and $\Omega$ resembles the parameter where $(\Omega \in\{0,1\})$ chosen to indicate the influence of the classification error over the extracted feature set. Then the movement of the features towards the optimal features in imitated based on the foraging strategy of manta rays.

The three different foraging strategies can be imitated to identify the best features from the three different feature set. The optimal features among the intensity, texture and shape feature sets are identified based on the chain, cyclone and somersault foraging strategies respectively. In the chain foraging, the optimal intensity feature is identified as the foraging point and the other secondary optimal intensity (LDP) features move towards it to form an optimal intensity feature subset. The position of every individual feature is identified and updated based on the following chain position update equation:

$x_{j}^{d}(t+1)=\left\{\begin{array}{l}x_{j}^{d}(t)+r_{a}\left(x_{b}^{d}(t)-x_{j}^{d}(t)\right)+\omega\left(x_{b}^{d}(t)-x_{j}^{d}(t)\right) \quad j=1 \\ x_{j}^{d}(t)+r_{a}\left(x_{j-1}^{d}(t)-x_{j}^{d}(t)\right)+\omega\left(x_{b}^{d}(t)-x_{j}^{d}(t)\right) \quad j=1,2, \ldots . n\end{array}\right.$

$\omega=2 * r_{a} \sqrt{\left|\log \left(r_{a}\right)\right|}$

The equation (39) signifies the movement of intensity features towards the optimal features where $x_{j}^{d}(t)$ indicates the position of $j^{\text {th }}$ individual in the $d^{\text {th }}$ space at time t, $\omega$ indicates the weight factor, $r_{a}$ is a random vector chosen between 0 and $1, x_{b}^{d}(t)$ is the best position identified by the foraging features where it is close to optimal one.The most optimal intensity features are identified from the chain foraging strategy.

Then the cyclone foraging strategy is imitated in this article to identify the optimal texture based feature(ULBP) set from the extracted texture features. The optimal texture feature that best defines the texture of the tumor is chosen the foraging point and the other texture features are set as the individuals that travels towards the optimum. The position of every foraging feature in the group is updated based on the following position update equation:

$x_{j}^{d}(t+1)= \begin{cases}x_{b}^{d}+r_{a}\left(x_{b}^{d}(t)-x_{j}^{d}(t)\right)+\varepsilon\left(x_{b}^{d}(t)-x_{j}^{d}(t)\right) & j=1 \\ x_{b}^{d}+r_{a}\left(x_{j-1}^{d}(t)-x_{j}^{d}(t)\right)+\varepsilon\left(x_{b}^{d}(t)-x_{j}^{d}(t)\right) & j=1,2, \ldots . . n\end{cases}$

$\varepsilon=2 e^{r_{a} \frac{T-t+1}{T}} \sin \left(2 \pi r_{a} 1\right)$

The above equation (42) signifies the weight factor $\varepsilon$ with $r_{a} 1$ indicating the random number between 0 and 1 and $\mathrm{T}$ indicating the maximum count of iterations. The cyclonic foraging strategy has the ability to find the global optimum among the features recognized.

Finally, the most unique and efficient somersault foraging strategy is imitated to identify the optimal shape feature (Gabor) set among the extracted shape features. The most optimal shape feature is identified more efficiently through this strategy and the position update takes place more frequently for every foraging feature which is depicted as follows:

$x_{j}^{d}(t+1)=x_{j}^{d}(t)+\xi\left(r_{a} 2 * x_{b}^{d}-r_{a} 3 * x_{j}^{d}(t)\right) j=1,2, \ldots . . n$

The somersault position update equation (43) introduces the somersault factor representing the range and it is set to $2, r_{a} 2$ and $r_{a} 3$ are two random numbers introduced that ranges between 0 and 1. For every movement, the best and optimal feature is identified based on the fitness evaluation between the identified solutions. The optimal fitness evaluation is represented as follows: 
$F E= \begin{cases}P^{\prime} ; & \text { if the position }\left(P^{\prime}\right) \text { is better than } P \\ P ; & \text { otherwise }\end{cases}$

The above equation is used by all the foraging strategies to identify the best feature out of the available intensity, texture and shape features. The output of the feature selection process will be the optimal features covering the features related to intensity, texture and shape enclosed in the MRI brain images. The high dimensional features are discarded efficiently through this feature selection strategy. The pseudo-code for the feature selection process is depicted in algorithm 1 .

Algorithm 1. MRFO for feature selection
Input: Population size n, stopping criteria T, search agents
Output: Optimal feature set
Step 1: Identify the fitness of every feature using (38)
Step 2: While $(i<T)$ do
Step 3: For $(i \rightarrow 1 b n)$ do
Step 4: Update random positions of every features
identified in search space
Step 5: For $(f \rightarrow$ intensity based) do
Step 6: Update position using (39)
Step 7: Evaluate fitness using (38)
Step 8: End for
Step 9: For $(f \rightarrow$ texture based) do
Step 10: Update position using (41)
Step 11: Evaluate fitness using (38)
Step 12: End for
Step 13: For $(f \rightarrow$ shape based) do
Step 14: Update position using (43)
Step 15: Evaluate fitness using (38)
Step 16: End for
Step 17: Compute overall fitness for the whole feature
sets using (44)
Step 18: End for
Step 19: End while
Step 20: Return optimal feature set P.

The selected features from the MRFO based feature selection process are then subjected to classification process where the grade of the tumors are categorized.

\subsection{Multi-grade Classification Using HDNN- AROA}

The selected features with reduced dimensionality are taken as the input of the classifier and the grade of the tumors are guessed based on certain prediction rules. For tumor grade classification, this article proposes the hybrid DNN-AROA technique to efficiently categorize the brain tumor with high classification accuracy. The classification strategy mainly aims to reduce the error rate with improved accuracy. The tumor classification depends on the appropriate features that are given as input that better signifies the growth of the tumors. The identified optimal features in the feature selection phase constitutes the most important characteristics that determines the tumor growth and these features are directly given as input to the DNN.

The DNN is an efficient neural network that classifies the tumors based on the grade more accurately than the other neural networks. The DNN is a feed-forward kind constituting one input layer, certain count of hidden layers and one output layer. The selected features are fed through the input layer in the DNN where the features are passed to the hidden layers to get classified. The hidden layer possess neurons that learn from the input features and classifies it accordingly. The DNN classifies the image based on the grade into two parts as low-level grade tumors (LGT) and high-level grade tumors (HGT). Initially, the input features that are fed to the neural network can be represented as follows:

$X=\left(x_{1}, x_{2}, \ldots \ldots x_{n}\right)$

The sequence of features that are optimally selected constitutes the major part in classification to accurately determine the grade of tumors. The input layer receives the input and passes it directly to the hidden layer without any changes where the classification procedure is started and performed. While processing, the input inside the hidden layer is mapped with certain weight $\left(w_{j}\right)$ and bias $\left(b_{k}\right)$ values that are randomly chosen for every associated hidden layers. The inputs for every sequential hidden layers can be framed as follows:

$h_{k}=\sum_{j} w_{j} x_{j}+b_{k}$

In the above mentioned equation, $w_{j}$ determines the weight value of the predecessor added while transmitting the input to the successor layer and $j$ indicates the count of hidden layers given as $k=1,2, \ldots \ldots . . l$. For every hidden layer, the weight and bias values are chosen randomly and updated with the input values for further processing in the succeeding hidden layers.

The hidden layers in the DNN are associated with activation function to decide on the input that has to be passed to the other hidden layer. Moreover, the training process can be efficiently boosted up with the activation function that helps the neural network to learn any kind of complex problems. There are many different activationslike ReLU, sigmoid, tanh, etc. are followed by various neural networks for various purposes. Out of all the activation functions, the ReLU has proved to improve the learning process by maintaining a balance even after 
several iterations. Thus the ReLU activation function has been chosen to trigger the output of every hidden layer to the next layer and also to improve the efficiency of learning. The overall output obtained from the hidden layer can be expressed based on the activation function as follows:

$H=f\left(h_{k}\right)$

Where $f\left(h_{k}\right)=\operatorname{Re} L U\left(h_{k}\right)$ symbolizes the influence of the activation function in processing the inputs. The overall output from the hidden layer comprises the weight and bias values added along with the activation functions. This output is transmitted to the output layer of DNN to achieve the classification output of brain tumor grade. In order to attain the classification output, the most preferred non-linear softmax activation function is introduced for obtaining the multi-grade classification output with high accuracy. The addition of softmax function helps in converting the input into a probability set for tumor classification. The overall classification output from the DNN can be demonstrated as follows:

$\sigma(X)_{k}=\frac{e^{X_{k}}}{\sum_{k=1}^{l} e^{X_{k}}}$

The output from the classifier will be a probability value indicating the tumor based on its growth. The output comprises two labels as LGT and HGT where the intensity measures of the tumor categorizes it into four different grades. The DNN comprises a loss function to indicate the deviation of the classification accuracyfrom the original input. Minimization of loss function is highly preferred to obtain a keen and accurate classification and to make the system more efficient. The error function of the DNN classifier can be given as:

$L_{f}=-\frac{1}{n} \sum_{j=1}^{n}\left(\left(y_{j} \log p_{j}\right)+\left(1-y_{j}\right) \log \left(1-p_{j}\right)\right)$

The DNN gets trained with vast and diverse range of input features and the classification accuracy determines the quality of the system in identifying the grade. But, the DNN comprises weight values that are changed randomly for every hidden layer which highly influences the output and the accuracy of classification. To avoid this problem, the article proposes a hybridized strategy where the optimization technique is used in updating the weight value of the DNN so that optimal weight value will be chosen with an aid of minimizing the error rate of classification.

\section{Updating weight based on AROA}

To update the weight value to improve the accuracy of the classification technique, the article imitates the rain's behavior where the radius of the droplets are considered for parameter tuning. The algorithm is imitated by taking the droplets as the search agents to identify the optimal weight factor. The droplets indicated as the search agents can be demonstrated as $\mathrm{O}=\left(d_{1}, d_{2}, \ldots . d_{r}\right)$. The variables identified in the droplet moves gradually downward and the limits of the variables are determined. The process is repeated for all the identified variables inside the droplet. Meanwhile, the cost function is updated by every single droplet. The radius of the droplet determines the weight factor of the hidden layer and the minimal cost will be taken as the optimal weight factor. An objective function is formulated to determine the optimality of the chosen weight factor. This can be given as follows:

Fit $=\min \left(L_{f}\right)$

For every new finding of the variables, the cost function is determines and the fitness function is evaluated based on the above Equation (50). The radius between the droplets influences the weight optimization based on the distance between them. When two droplets with different radii $v_{1}$ and $v_{2}$ are located very close to each other, these two can combine to form a large droplet with radius $R$ as follows:

$R=\left(v_{1}^{n}+v_{2}^{n}\right)^{\frac{1}{n}}$

While $\mathrm{n}$ indicating the count of variables idealized in each droplet. Similarly, the droplet might face changes based on the absorption by the soil and the properties of the soil. Assuming that the droplet $v_{1}$ is absorbed by the soil, the droplet radius can be updated as follows:

$R=\left(\lambda v_{1}^{n}\right)^{\frac{1}{n}}$

In the above Equation (53) $\lambda$ indicates the absorption rate of the droplet for every iterations. The search agents perform the search to find the optimal weight out of all the randomly chosen weight factors while classification. For every new finding by the droplet, the fitness function is evaluated to identify the optimal weight to minimize the error rate and to achieve better and efficient classification accuracy. The overall algorithm for updating the weight factor is depicted in algorithm 2 .

Thus the brain tumor gets classified more accurately based on the grades. The output of the classification process labels the trained images into LGT and HGT where the four different grades of the tumors are identified. Moreover, the DNN with the help of AROA produces more accurate output with reduced error. 


\section{Algorithm 2: AROA for weight update}

Input:weight factor to be tuned $\left(w_{j}\right)$, maximum count of iterations $\left(\right.$ Max $\left._{i t r}\right)$, initial radius of all the droplets, $\lambda$, variable sizes, count of joined droplets

Output: Optimal weight factor

Step 1: Initialize the population size, droplet radius and positions

Step 2: Evaluate for fitness for all the identified droplets using (50) and sort the search agents based on the weight factor

Step 3: While $\left(i<\right.$ Max $\left._{i t r}\right)$ do

Step 4: For $\left(i \rightarrow 1\right.$ to $\left.d_{r}\right)$

Step 5: Determine the movement of the variables

Step 6: Evaluate the new positions identified using (50)

Step 7: If $\left(\right.$ New $_{p o s}<$ Old $\left.p o s\right)$ do

Step 8: Choose $\mathrm{New}_{\text {pos }}$

Step 9: While $\left(w_{j}\right.$ reduces $)$

Step 10: Move the search agents in the same direction

Step 11: Compute $\lambda$ and reduce the size of search agent

Step 12: Compute the new size of the droplets

Step 13: End while

Step 14: End for

Step 15: Neglect the worst search agents

Step 16: Generate new search agents to identify more optimal weight factor

Step 17: End while

Step 18: Determine the search agents identified with cost

Step 19: Return(optimal weight)

\section{Simulation Analysis}

This section presents the simulation analysis and results of the proposed approach to prove the performance efficiency in comparison with the other existing state-ofthe-art approaches. The existing techniques like Gabormodulated CNN ${ }^{[25]}, \mathrm{SVM}+\mathrm{D}-\mathrm{SEG}{ }^{[26]}$, RFE-SVM ${ }^{[27]}$, $\mathrm{mRMR}+\mathrm{SVM}{ }^{[28]}$, logistic regression ${ }^{[29]}$, ensemble method ${ }^{[30]}$, U-net $\mathrm{NN}^{[31]}$ and hybrid model ${ }^{[32]}$ has been chosen for comparison for the metrics such as precision, recall, accuracy and Dice Similarity Coefficient (DSC). The detailed description of the datasets used and the experimental scenarios are presented in the next part and the performance analysis and comparison are presented in the upcoming sections.

\subsection{Simulation Scenario}

The proposed technique used the images obtained from two different datasets relevant to brain tumor such as Radiopaedia dataset and Brain Tumor detection dataset (BTS). Many different experiments are carried out to ensure the accuracy and efficiency of the proposed approach against the other compared techniques. Among the dataset images, $80 \%$ of the total images are considered for training and the remaining $20 \%$ are used for testing. The implementation of the work is carried out in Python.

\section{Radiopaedia Dataset}

The radiopaedia dataset is commonly used for the training and testing purposes for brain tumor grade classification. The images in this dataset are classified into four different grades that helps in efficient classification of any kind of brain tumor depending on the growth of the tumor. This dataset comprises $121 \mathrm{MR}$ brain tumor images that are classified into four major grades like meningiomas, gliomas grade II, gliomas grade III and glioblastomas. The tumor images are classified and labelled based on the guidelines described by WHO.

\section{BTS Dataset}

The BTS dataset is more appropriate for brain tumor grade classification and it covers grade images such as meningioma, glioma and pituitary tumor. These grade images are used along with the radiopaedia images in the proposed technique for efficient classification. This dataset comprises 3064 T1-weighted MR images with high contrast derived from 233 patients. The resolution of each of the MR images range into $512 \times 512$ with $0.49 \times 0.49$ pixel size. The thickness of the image slice is $6 \mathrm{~mm}$ and the gap between every image slice is identified as $1 \mathrm{~mm}$.

\subsection{Performance Metrics}

This part covers the performance metrics considered for evaluation of the proposed approach against the other approaches. The major performance metrics such as precision, recall, accuracy and DSCare considered for evaluation. The analysis are noted for all the other compared techniques with the proposed HDNN-AROA technique to verify the efficiency.

(i)Precision: Precision is the most significant metric considered to evaluate the classification approaches. It defines the rate of acceptably classified images based on the label i.e. the total count of true positives. It is obtained as the sum of exactly labelled images belonging to a particular class divided by the total count of images classified into the given label. The formulation can be given as follows:

$P_{n}=\frac{T p}{T p+F p}$

In the above Equation (52), $T_{p}$ denotes the true positive 
values and $F_{p}$ denotes the positive values. It helps in determining the properly classified images and thereby verifies the accuracy of the overall system

(ii) Recall:Recall is another important metric that helps in identifying the overall system accuracy. It can be defined as the total count of exactly classified images out of the total count of images that should have been classified for a particular label. The formulation can be given as follows:

$R_{l}=\frac{T p}{T p+F n}$

Where $F_{n}$ signifies the positives which means that the actual value is true but the result obtained is . The error in classification can be identified through this metric.

(iii)Accuracy: Accuracy is the most important metric that evaluates the efficiency and the improvement rate of any proposed classification strategy compared with other approaches. The accuracy obtained should be eventually high than any other approaches taken for comparison to prove the efficiency. The formulation for accuracy can be given as follows:

$A_{y}=\frac{T p+T n}{T p+T n+F p+F n}$

The accuracy plays a vital role in determining the performance of the classification technique in classifying the images into HGT and LGT. All the images are subjected to the classification to identify the intensity of the tumor and accurate classification empowers the technique to outperform the other techniques.

(iv)DSC: DSC is a statistical measure to identify the similarity between the classified images. The overlap between the two images can be identified using the following formula:

$D S C=\frac{2 T p}{2 T p+F p+F n}$

DSC metric relates both the precision and recall measures and helps in identifying the accuracy of the system. The highest value of DSC can be 1 indicating maximum precision and recall and the lowest value can be 0 indicating either the precision or the recall is 0 .

All these metrics are computed for the two different datasets used and the method is compared with the existing approaches to evaluate the performance of the approach with different images.

\subsection{Performance Analysis}

This part covers the comparative analysis of the proposed classification technique against other approaches. From the overall simulations performed, it has been identified that the proposed approach worked well in classifying the images accurately based on the growth of the tumors. The images form the two different datasets are used to evaluate the proposed approach and the classification strategy classifies the acquired images into HGT and LGT depending on the tumor growth. The simulations proved the efficiency and the improvement in the accuracy rate of the system against the other approaches. Moreover, the system clearly implied the efficiency of the system in appropriate feature selection that accurately determines the growth of the tumor. The performance evaluation of the proposed approach on two different datasets are as follows:

\section{Analysis of the proposed approach on Radiopaedia Dataset}

The images of the radiopaedia dataset are used where the $80 \%$ are used for training the classifier and the remaining $20 \%$ are taken for testing. For the images, the performance evaluation is done for the metrics such as precision, accuracy, recall and DSC. The graphs are depicted to demonstrate the performance improvement rate of the proposed approach against the other approaches. The HDNN-AROA classifier has been used for classification of the acquired images and the efficiency of the system has been improved by the addition of the optimizer. The learning rate of the classifier has been improved and hence efficient classification has been provided for all the images of the dataset.This is validated with the help of the performance metrics and the hybridization of the optimizer with the classifier showed better significance than the other approaches. The graphical representation of the evaluation metrics are presented as follows:

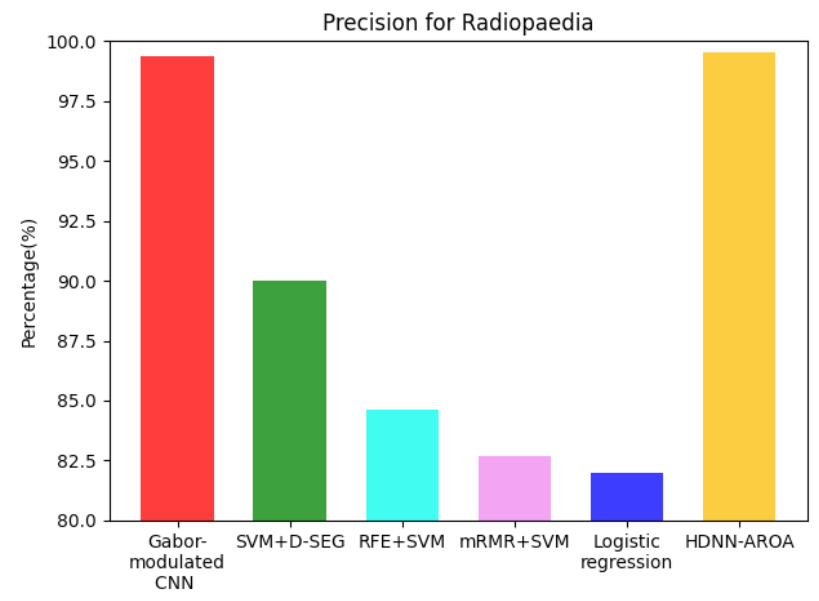

Figure 2. Graphical Representation of precision metric comparison for radiopaedia dataset 
The graphical representation in Figure 2 depicts the performance of the proposed approach in terms of precision against the other approaches. From the graph it has been identified that the proposed approached showed high precision range than the other approaches. Gabor-modulated $\mathrm{CNN}$ technique showed a high precision value that is closely equal to the proposed strategy. The overall precision range of the proposed strategy for the radiopaedia dataset is found to be $99.6 \%$ whereas the gabor-modulated CNN strategy showed 99.77 precision rate.

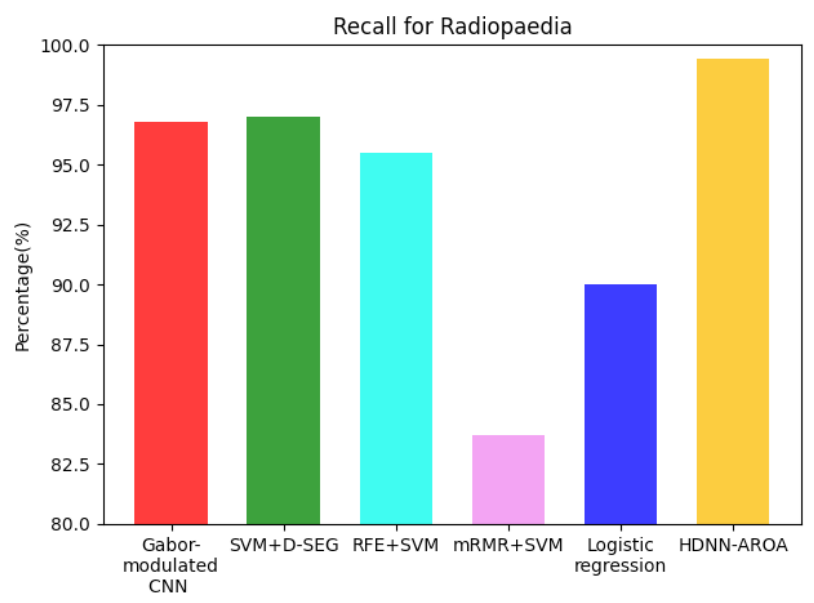

Figure 3. Graphical Representation of recall metric comparison for radiopaedia dataset

From the graphical representation depicted in Figure 3, it has been identified that the proposed technique showed a higher recall value than the other techniques compared with it. In terms of recall, the gabor-modulated $\mathrm{CNN}$ and SVM+D-SEG showed a close relevance with the proposed technique but the recall value for the proposed technique is 99.38\% whereas the recall rates for gabor-modulated CNN and SVM+D-SEG are $96.39 \%$ and $90 \%$ respectively.

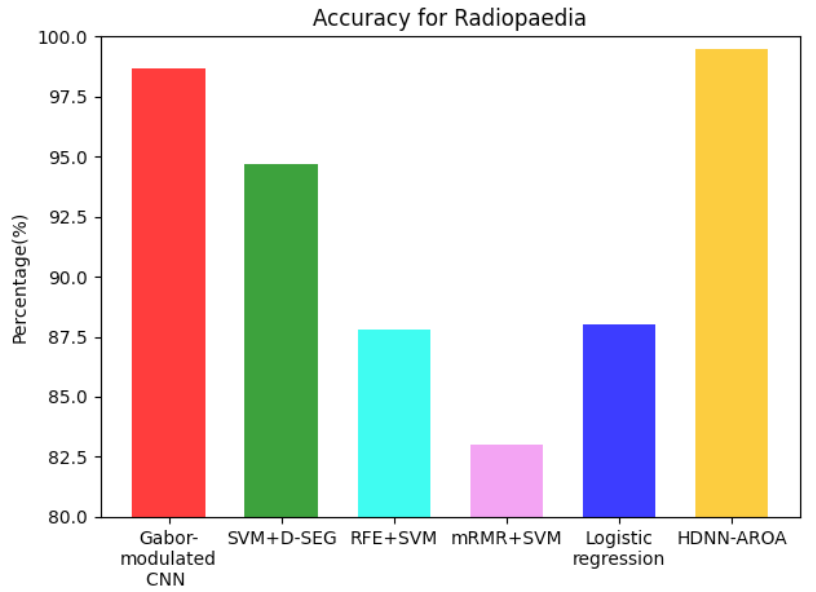

Figure 4. Graphical Representation of accuracy metric comparison for radiopaedia dataset
In terms of accuracy in classification, the proposed technique outperformed the other compared approaches. This is depicted in Figure 4 where the proposed technique showed a higher accuracy rate which is more comparable to the gabor-modulated CNN approach. The accuracy rate of the proposed technique is $99.5 \%$ and for gabormodulated CNN, the accuracy rate is $98.68 \%$.

Table 1. Summarization of the performance metrics for classification for Radiopaedia

\begin{tabular}{cccc}
\hline Classification Techniques & Precision (\%) & Recall (\%) & Accuracy (\%) \\
\hline Gabor Modulated CNN & 96.77 & 96.39 & 98.68 \\
SVM+D-SEG & 97 & 90 & 94.7 \\
RFE+SVM & 95.5 & 84.6 & 87.8 \\
mRMR+SVM & 83.7 & 82.7 & 83 \\
Logistic Regression & 90 & 82 & 88 \\
Proposed & 99.6 & 99.38 & 99.5 \\
\hline
\end{tabular}

The summarization of the values obtained for the performance evaluation of the proposed technique with other techniques for radiopaedia dataset is given in Table 1. From the graphs, it has been clearly identified that the proposed technique showed a higher classification accuracy than the other techniques. The gabor-modulated CNN technique also showed a higher and a better precision value and the recall rate was also found to be good. The technique more or less showed an equivalent accuracy rate to the proposed technique.

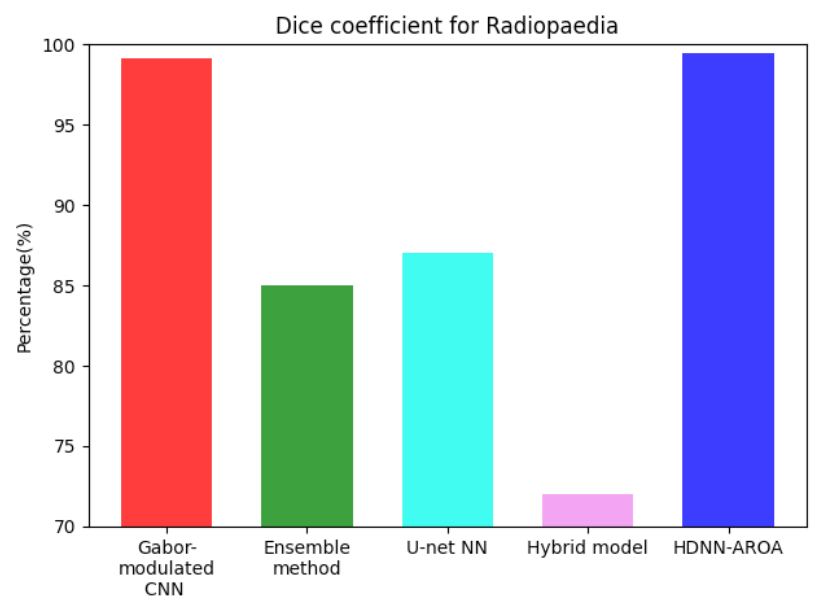

Figure 5. Graphical Representation of DSC metric comparison for radiopaedia dataset

The DSC of the proposed technique compared with the other techniques are represented graphically in Figure 5. From the graph, it has been identified that the proposed technique showed a better DSC rate than the other techniques. The gabor-modulated CNN is the only compared technique that showed a significant relevance to the proposed technique in DSC rate. The DSC rate of the 
proposed technique is $99.49 \%$ whereas the DSC rate of the Gabor-modulated CNN is found to be $99.10 \%$.

Table 2. Summarization of the DSC performance metric for Radiopaedia

\begin{tabular}{cc}
\hline Classification Techniques & DSC (\%) \\
\hline Gabor Modulated CNN & 99.10 \\
Ensemble Method & 85 \\
U-net NN & 87 \\
Hybrid Model & 72 \\
Proposed & 99.49 \\
\hline
\end{tabular}

\section{Analysis of the proposed approach on BTS Dataset}

The images acquired from the BTS dataset are used for classification where the $80 \%$ of the images are used for training and the remaining $20 \%$ are used for testing. The same approaches are used for performance evaluation of the proposed approach against the other approaches. The graphical representation of the different performance metrics and the values obtained indicating the performance improvement are as follows:

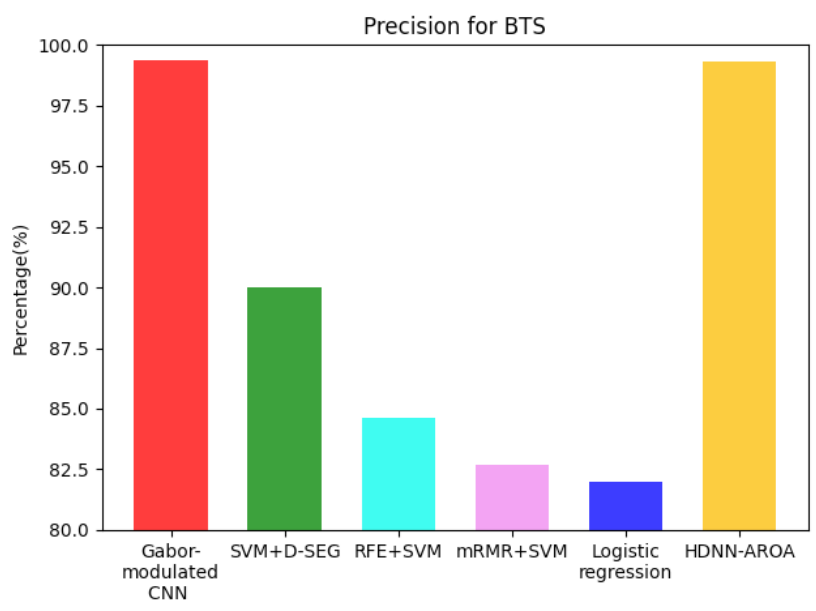

Figure 6. Graphical Representation of precision metric comparison for BTS dataset

The graphical representation in Figure 6 indicates the precision rate of the proposed approach with other approaches. From the graph, it has been identified that the proposed approach has shown higher precision rate than the other techniques. The gabor-modulated CNN showed an even better precision rate than the proposed approach. The precision rate of the proposed approach is found to be 99.39\% whereas for gabor-modulated $\mathrm{CNN}$ it is found to be $99.5 \%$.

Figure 7 depicts the graphical representation of the recall rate of the proposed approach compared with other approaches. From the graph, it has been identified that the proposed approach showed a higher recall rate than the other techniques. The gabor-modulated CNN and $\mathrm{SVM}+\mathrm{D}-\mathrm{SEG}$ techniques also showed a better recall rate when compared with the other approaches. The recall rate of the proposed technique for BTS dataset is found to be $99.2 \%$.

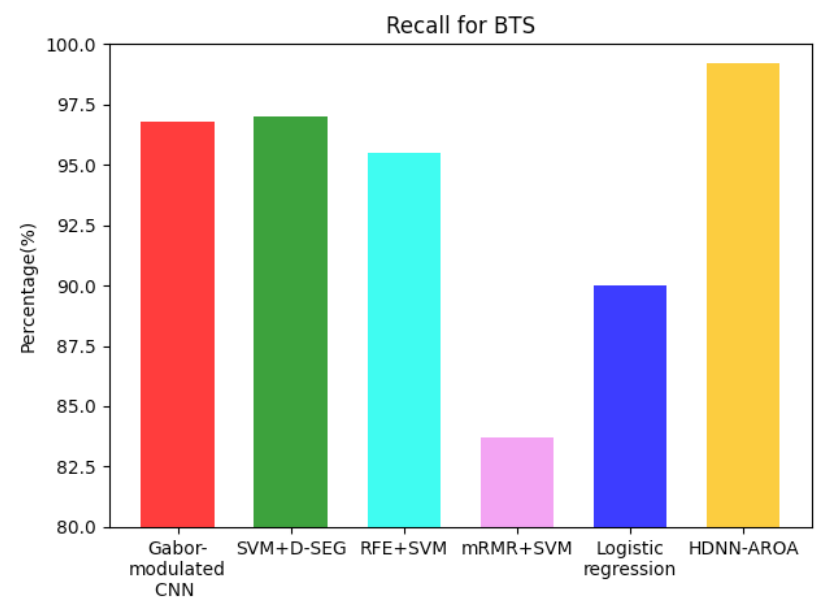

Figure 7. Graphical Representation of recall metric comparison for BTS dataset

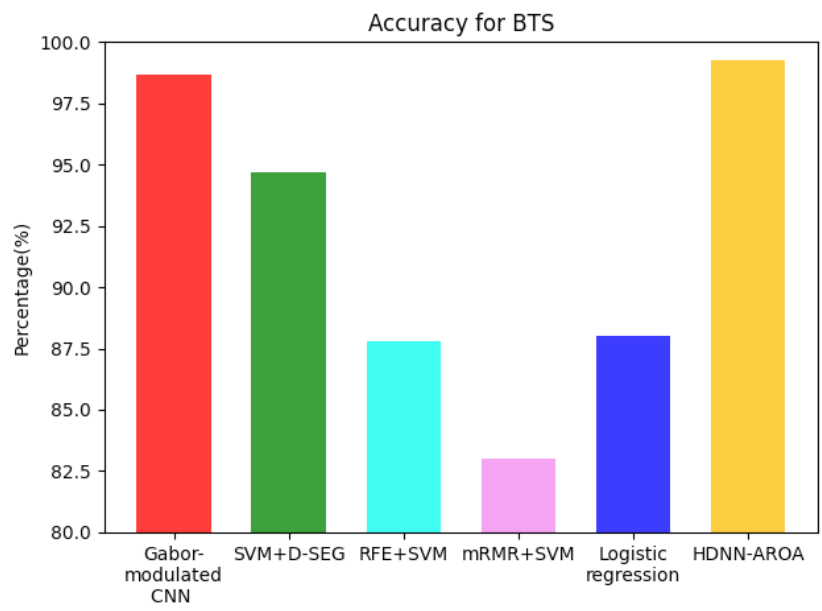

Figure 8. Graphical Representation of accuracy metric comparison for BTS dataset

Figure 8 depicts the accuracy rate of the proposed technique for BTS dataset compared with other approaches. From the graph, it is clearly identified that the proposed technique outperformed the other techniques in terms of accuracy. The overall accuracy rate of the proposed technique is found to be $99.27 \%$ which is significantly higher than any other techniques.

Table 3 summarizes the performance values obtained through simulation of the techniques with BTS dataset. It is clear from the table that the proposed approach showed a higher accuracy rate than any other techniques. The accuracy of the system is highly important to determine 
the classification efficiency of the system.

Table 3. Summarization of the performance metrics for classification for BTS dataset

\begin{tabular}{cccc}
\hline Classification Techniques & Precision (\%) & Recall (\%) & Accuracy (\%) \\
\hline Gabor Modulated CNN & 96.77 & 99.39 & 98.68 \\
SVM+D-SEG & 97 & 90 & 94.7 \\
RFE+SVM & 95.5 & 84.6 & 87.8 \\
mRMR+SVM & 83.7 & 82.7 & 83 \\
Logistic Regression & 90 & 82 & 88 \\
Proposed & 99.39 & 99.2 & 99.27 \\
\hline
\end{tabular}

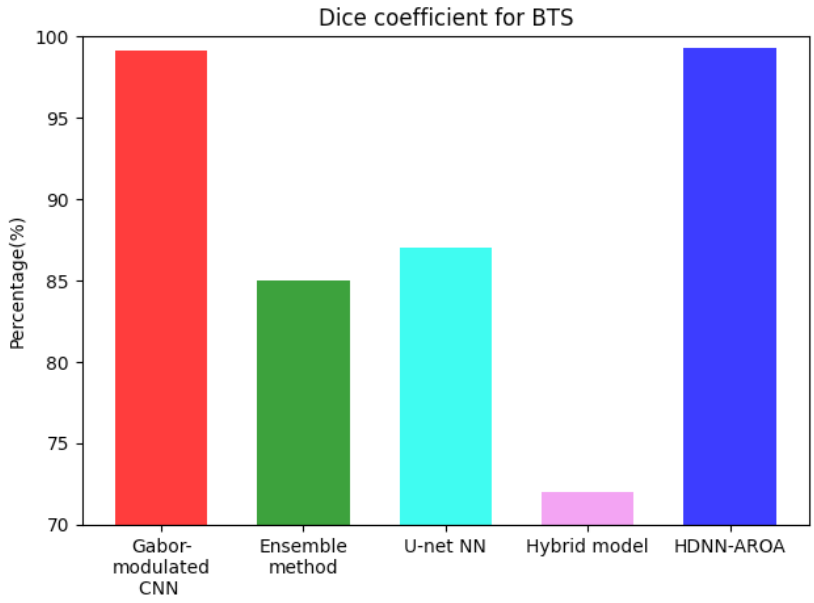

Figure 9. Graphical Representation of DSC metric comparison for BTS dataset

Figure 9 depicts the graphical representation of the proposed approach in terms of DSC for the BTS dataset. From the graph, it is noted that the proposed technique and gabor-modulated CNN techniques showed a higher DSC values than the other techniques. The DSC value of the proposed technique for BTS dataset is $99.28 \%$ and that for gabor-modulated $\mathrm{CNN}$ is $99.10 \%$.

Table 4. Summarization of the DSC performance metric for classification for BTS dataset

\begin{tabular}{cc}
\hline Classification Techniques & DSC (\%) \\
\hline Gabor Modulated CNN & 99.10 \\
Ensemble Method & 85 \\
U-net NN & 87 \\
Hybrid Model & 72 \\
Proposed & 99.28 \\
\hline
\end{tabular}

Table 4 illustrates the summarization of the DSC obtained trough proposed technique for BTS dataset. From the table, it is clear that the proposed technique showed a higher DSC value of $99.28 \%$ than any other proposed techniques. The existing techniques compared with the proposed technique almost showed similar results for both the datasets whereas the proposed technique showed higher accuracy in classifcation of radiopaedia images. The precision and recall values for both the datasets are almost similar but the accuracy values had a mild difference for the proposed technique.

\section{Training Dataset}
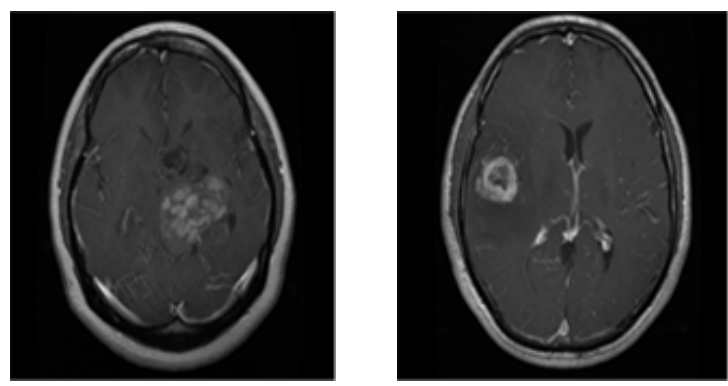

Testing Dataset
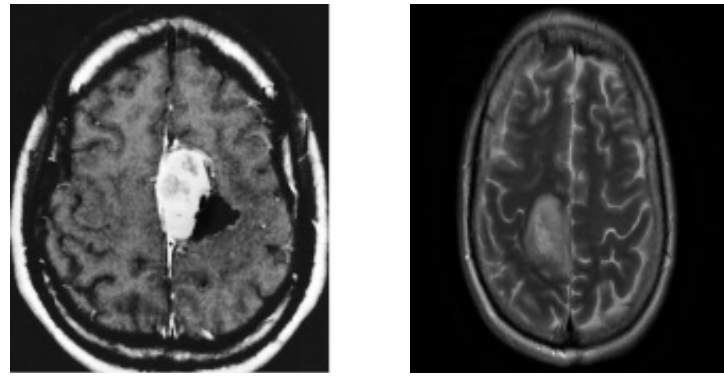

Figure 10. An example of the training and testing images acquired from the radiopaedia dataset

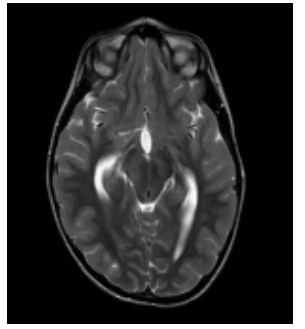

Glioma

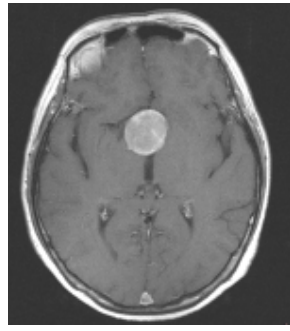

Pituiatry

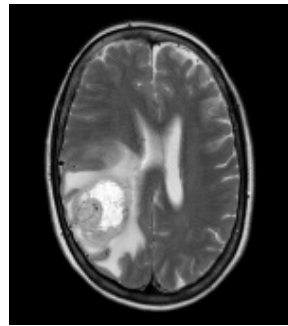

Glioblastoma

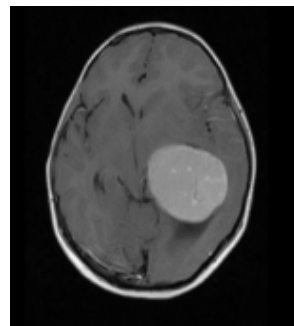

Meningioma
Figure 11. The sample of the tumor grade images acquired from the radiopaedia and BTS datasets

An example of the training and testing images taken from the radiopaedia dataset are depicted in Figure 10. From the figure, the presence of the tumor on the brain can be identified. Both the training and testing datasets 
consists of tumor regions and the proposed technique works on classifying the images based on the tumor growth. The images are classified into HGT or LGT depending on the growth and the HGT covers grade I and II and the LGT demonstrates the grade III and grade IV tumors. Figure 11 depicts the four different grades of tumors taken from the two different datasets. The location of the tumor on the brain region is identified and then the basic features are extracted and then classified accordingly.

\section{Conclusions}

Multi-grade brain tumor classification plays a significant role in the medical field to identify the growth and seriousness of the tumors in the brain. The MRI images can be used to identify the tumors and to present a detailed study on it. This paper presents a solution for multi-grade brain tumor classification with higher accuracy. The images acquired from the radiopaedia and BTS datasets are used in the proposed approach and those images are subjected to pre-processing initially. Then, the piFCM clustering method is used for image segmentation to reduce the uncertainty. After segmentation, the feature extraction process is proposed to derive the most important intensity, texture and shape features from the images. After extraction, the major features are selected using the MRFO algorithm based on the foraging strategies of manta rays. Finally the images are subjected to classification where the technique proposed the HDNNAROA technique to classify the images based on the grades. The technique classified the images into HGT and LGT based on the tumor growth. The proposed technique showed a higher accuracy rate for both the datasets when compared with the other techniques. The analysis of the proposed technique proved that the technique is more efficient than any other existing techniques and the precision, recall, accuracy and DSC for radiopaedia dataset are found to be $99.6 \%, 99.38 \%, 99.5 \%$ and $99.49 \%$ and for BTS dataset are found to be $99.39 \%$, $99.2 \%, 99.27 \%$ and $99.28 \%$ respectively.

\section{References}

[1] Deepak, S., Ameer, P.M., 2019 Aug. 1. Brain tumor classification using deep CNN features via transfer learning. Computers in biology and medicine. 111, 103345.

[2] Prabhu, L.A., Jayachandran, A., 2018 Dec. Mixture model segmentation system for parasagittal meningioma brain tumor classification based on hybrid feature vector. Journal of medical systems. 42(12), 1-6.
[3] Ryall, S., Tabori, U., Hawkins, C., 2020 Dec. Pediatric low-grade glioma in the era of molecular diagnostics. Acta neuropathologica communications. 8(1), $1-22$.

[4] Maekawa, T., Hori, M., Murata, K., Feiweier, T., Kamiya, K., Andica, C., Hagiwara, A., Fujita, S., Koshino, S., Akashi, T., Kamagata, K., 2020 Oct. 1. Differentiation of high-grade and low-grade intra-axial brain tumors by time-dependent diffusion MRI. Magnetic Resonance Imaging. 72, 34-41.

[5] Aboelenein, N.M., Songhao, P., Koubaa, A., Noor, A., Afifi, A., 2020 May 29. HTTU-Net: Hybrid Two Track U-Net for automatic brain tumor segmentation. IEEE Access. 8, 101406-15.

[6] Pang, S., Du, A., Orgun, M.A., Yu, Z., Wang, Y., Wang, Y., Liu, G., 2020 Sep. CTumorGAN: a unified framework for automatic computed tomography tumor segmentation. European journal of nuclear medicine and molecular imaging. 47(10), 2248-68.

[7] Zhou, Z., He, Z., Jia, Y., 2020 Aug. 18. AFPNet: A $3 \mathrm{D}$ fully convolutional neural network with atrous-convolution feature pyramid for brain tumor segmentation via MRI images. Neurocomputing. 402, 235-44.

[8] Dequidt, P., Bourdon, P., Ahmed, O.B., Tremblais, B., Guillevin, C., Naudin, M., Fernandez-Maloigne, C., Guillevin, R., 2019 Oct. 17. Recent advances in Glioma grade classification using machine and deep learning on MR data. In 2019 Fifth International Conference on Advances in Biomedical Engineering (ICABME) IEEE. pp. 1-4.

[9] Ali, M.B., Gu, I.Y., Berger, M.S., Pallud, J., Southwell, D., Widhalm, G., Roux, A., Vecchio, T.G., Jakola, A.S., 2020 July. Domain Mapping and Deep Learning from Multiple MRI Clinical Datasets for Prediction of Molecular Subtypes in Low Grade Gliomas. Brain Sciences. 10(7), 463.

[10] Menze, B., Isensee, F., Wiest, R., Wiestler, B., Maier-Hein, K., Reyes, M., Bakas, S., 2020 Dec. 2. Analyzing Magnetic Resonance Imaging Data from Glioma Patients using Deep Learning. Computerized Medical Imaging and Graphics. 101828.

[11] Minaee, S., Boykov, Y., Porikli, F., Plaza, A., Kehtarnavaz, N., Terzopoulos, D., 2020 Jan. 15. Image segmentation using deep learning: A survey. arXiv preprint arXiv:2001.05566.

[12] Joardar, B.K., Jayakodi, N.K., Doppa, J.R., Li, H., Pande, P.P., Chakrabarty, K., 2020 Mar. 9. GRAMARCH: A gpu-reram based heterogeneous architecture for neural image segmentation. In2020 Design, Automation \& Test in Europe Conference \& 
Exhibition (DATE). IEEE. pp. 228-233.

[13] Kurc, T., Bakas, S., Ren, X., Bagari, A., Momeni, A., Huang, Y., Zhang, L., Kumar, A., Thibault, M., Qi, Q., Wang, Q., 2020. Segmentation and classification in digital pathology for glioma research: Challenges and deep learning approaches. Frontiers in neuroscience. 14.

[14] Udendhran, R., Balamurugan, M., Suresh, A., Varatharajan, R., 2020 July 1. Enhancing image processing architecture using deep learning for embedded vision systems. Microprocessors and Microsystems. 76, 103094.

[15] Zhang, H.M., Dong, B., 2020 Jan. A review on deep learning in medical image reconstruction. Journal of the Operations Research Society of China. 10, 1-30.

[16] Narmatha, C., Eljack, S.M., Tuka, A.A., Manimurugan, S., Mustafa, M., 2020 Aug. A hybrid fuzzy brain-storm optimization algorithm for the classification of brain tumor MRI images. Journal of Ambient Intelligence and Humanized Computing. 14, 1-9.

[17] Kumar, R.L., Kakarla, J., Isunuri, B.V., Singh, M., 2021 Jan. Multi-class brain tumor classification using residual network and global average pooling. Multimedia Tools and Applications. 15, 1-0.

[18] Ayadi, Wadhah, Wajdi Elhamzi, Imen Charfi, Mohamed Atri, 2021. Deep CNN for Brain Tumor Classification. Neural Processing Letters. pp. 1-30.

[19] Sasank, V.V., Venkateswarlu, S., 2021 Jan. Brain tumor classification using modified kernel based softplus extreme learning machine. Multimedia Tools and Applications. 15, 1-22.

[20] Mzoughi, H., Njeh, I., Wali, A., Slima, M.B., BenHamida, A., Mhiri, C., Mahfoudhe, K.B., 2020 Aug. Deep multi-scale 3D convolutional neural network (CNN) for MRI gliomas brain tumor classification. Journal of Digital Imaging. 33, 903-15.

[21] Salehi, Hadi, Javad Vahidi, Thabet Abdeljawad, Aziz Khan, Seyed Yaser Bozorgi Rad, 2020. A SAR Image Despeckling Method Based on an Extended Adaptive Wiener Filter and Extended Guided Filter. Remote Sensing. 12(15), 2371.

[22] Shaukat, Furqan, Gulistan Raja, Rehan Ashraf, Shehzad Khalid, Mudassar Ahmad, Amjad Ali, 2019. Artificial neural network based classification of lung nodules in CT images using intensity, shape and texture features. Journal of Ambient Intelligence and Humanized Computing 10. 10, 4135-4149.

[23] Shaukat, Furqan, Gulistan Raja, Rehan Ashraf, Shehzad Khalid, Mudassar Ahmad, Amjad Ali, 2019. Artificial neural network based classification of lung nodules in CT images using intensity, shape and texture features. Journal of Ambient Intelligence and Humanized Computing. 10(10), 4135-4149.

[24] Mohanaiah, P., Sathyanarayana, P., GuruKumar, L., 2013. Image texture feature extraction using GLCM approach. International journal of scientific and research publications. 3(5), 1-5.

[25] Singh, Rahul, Aditya Goel, Raghuvanshi, D.K., 2020. Computer-aided diagnostic network for brain tumor classification employing modulated Gabor filter banks. The Visual Computer. 1-15.

[26] Jones, Timothy, L., Tiernan, J., Byrnes, Guang Yang, Franklyn, A., Howe, B., Anthony Bell, Thomas, R., Barrick, 2015. Brain tumor classification using the diffusion tensor image segmentation (D-SEG) technique. Neuro-oncology. 17(3), 466-476.

[27] Zacharaki, Evangelia I., Sumei Wang, Sanjeev Chawla, Dong Soo Yoo, Ronald Wolf, Elias R. Melhem, Christos Davatzikos, 2009. Classification of brain tumor type and grade using MRI texture and shape in a machine learning scheme." Magnetic Resonance in Medicine: An Official Journal of the International Society for Magnetic Resonance in Medicine. 62(6), 1609-1618.

[28] Soltaninejad, Mohammadreza, Guang Yang, Tryphon Lambrou, Nigel Allinson, Timothy L. Jones, Thomas R. Barrick, Franklyn A. Howe, Xujiong Ye, 2017. Automated brain tumour detection and segmentation using superpixel-based extremely randomized trees in FLAIR MRI. International journal of computer assisted radiology and surgery. 12(2), 183-203.

[29] Hsieh, Kevin Li-Chun, Chung-Ming Lo, Chih-Jou Hsiao, 2017. Computer-aided grading of gliomas based on local and global MRI features." Computer methods and programs in biomedicine. 139, 31-38.

[30] Cabezas, Mariano, Sergi Valverde, Sandra GonzálezVillà, Albert Clérigues, Mostafa Salem, Kaisar Kushibar, Jose Bernal, Arnau Oliver, Xavier Lladó, 2018. Survival prediction using ensemble tumor segmentation and transfer learning. arXiv preprint arXiv: 1810.04274 .

[31] Caver, Eric, Chang, L., Zong, W.W., Dai, Zh.Zh., Wen, N., 2018. Automatic brain tumor segmentation using a U-net neural network." In Pre-Conference Proceedings of the 7th MICCAI BraTS Challenge. pp. 63-73.

[32] Meier, H., Raphael, Stefan Bauer, Johannes Slotboom, Roland Wiest, Mauricio Reyes, 2013. A hybrid model for multimodal brain tumor segmentation." Multimodal Brain Tumor Segmentation. 31, 31-37. 